\title{
Sestamibi-Negative Patients: To Operate or Image?
}

\author{
Carrie C. Lubitz, MD, MPH ${ }^{1}$ and Herbert Chen, $\mathrm{MD}^{2}$ \\ ${ }^{1}$ Endocrine Surgery Unit, Department of Surgery, Massachusetts General Hospital, Harvard Medical School, Boston, MA; \\ ${ }^{2}$ Department of Surgery, UW School of Medicine and Public Health, Madison, WI
}

Parathyroidectomy is accepted as the most durable and cost-effective treatment for patients with primary hyperparathyroidism, with reported cure rates over $97 \% .^{1,2}$ Surgical cure prevents-and in some cases reversescomplications such as frequency of nephrolithiasis, osteoporosis, cardiovascular disease, and neurocognitive symptoms. ${ }^{3-7}$ The large prevalence of single-gland disease has led to the practice shift to focused parathyroidectomy, requiring preoperative localization. ${ }^{8}$ Moreover, most patients in the United States present with mild or asymptomatic disease with minor biochemical abnormalities, making management increasingly challenging.

In this study, Benzon and colleagues address the particular challenge of sestamibi-negative localization in patients with primary hyperparathyroidism and report the experience of a single highly reputable academic institution. Among the strengths of the study are the prospective collection of data and the large sample size. Of the 2681 patients who were referred for surgery in their cohort, 136 of patients $(5.7 \%)$ had sestamibi-negative imaging. The authors found that patients who localized with sestamibi had a $97 \%$ 6-month cure rate compared to $90 \%$ in those whose disease was not localized preoperatively, comparable to rates in the literature. Moreover, the study affirms the known correlation between sestamibi-negative imaging and multiglandular disease ( $32 \%$ vs. $13 \%$ in localized cases).

The authors discuss the clinical dilemma presented to physicians in sestamibi-negative cases and include observation as well as additional imaging and surgery as a consideration in asymptomatic patients. One should be cautious, however, about the suggestion to consider observing patients whose disease is not localized. If one

(C) Society of Surgical Oncology 2012

Published Online: 24 April 2012

H. Chen, MD

e-mail: chen@surgery.wisc.edu were to assess the appropriateness of our current standard of care, specifically criteria for parathyroidectomy in asymptomatic patients, a prospective trial with clearly defined inclusion criteria and a comparable control group (i.e., sestamibi-negative patients who are observed) would be needed. ${ }^{9}$ Until evidence contradicts our current understanding, practice ought to be based on the best available data and guidelines. ${ }^{5,9}$ It should be stressed, as the authors do, that the indication for surgery is the morbidity associated with the disease (i.e., continued bone loss, nephrolithiasis) and not the results of imaging. Frequently, patients labeled asymptomatic have improvement in subtle neurocognitive symptoms after parathyroidectomy. ${ }^{7}$ If it is possible that referring physicians are less likely to refer asymptomatic patients for surgery, as the authors suggest, perhaps our focus should be on education of the benefits of parathyroidectomy.

The question to be answered is not whether to operate or not, as the cure rate is still excellent in poorly localized cases, but whether to obtain further localization studies or to proceed directly to surgery. Although preoperative localization strategies vary among institutions on the basis of availability and clinician preferences, adjunctive or alternative localization modalities can be considered. Although the authors report minimal benefit of adjunctive ultrasound (US) in their series, others have found US to be highly sensitive and cost-effective. ${ }^{10,11}$ US is increasingly performed in the surgeon's office, costs less than other modalities, and has the advantage of imaging the thyroid simultaneously before surgery. Additionally, 4DCT scanning is a novel imaging modality that has shown improved sensitivity over ultrasound and sestamibi and should be considered, when available, for initial localization or in patients whose traditional imaging results are negative. ${ }^{10}$ 4DCT is particularly helpful in identifying ectopic glands, another frequent cause of surgical failure. ${ }^{12}$ Lastly, radioguided parathyroidectomy is an another useful modality 
that has been shown in a large series to be equally effective in patients with negative preoperative sestamibi findings. ${ }^{13}$ Adjunctive imaging with US or 4DCT along with intraoperative PTH could permit focused parathyroidectomy in some sestamibi-negative cases.

Alternatively, a viable but less cost-effective option is proceeding directly with four-gland exploration. Although the evolution to minimally invasive parathyroidectomy over the past few decades has led to less experience and, perhaps, comfort with four-gland exploration, it remains an effective option, especially in patients at high risk for multigland or ectopic disease, such as sestamibi-negative cases. Moreover, experience with four-gland exploration, multigland disease, and the nuances of intraoperative PTH use are imperative to the successful treatment of these patients with poorly localized disease.

In conclusion, Benzon and colleagues report an increasingly common clinical challenge in patients presenting with primary hyperparathyroidism. Importantly, the take-home points underscored by the authors are the following: (1) patients with poorly localized disease are best treated at a referral center with experience endocrine surgeons, and (2) informing patients and referring physicians about the higher rate of failure in sestamibi-negative patients is warranted. Surgical treatment remains indicated, with excellent cure rates even in patients with poorly localized disease. Whether to proceed with further localization studies, including US, 4DCT, and radioguidance, versus proceeding directly to surgery will vary on the basis of institutional and surgeon practices, availability of alternative imaging, cost, and ultimately patient preference.

\section{REFERENCES}

1. Siperstein A, Berber E, Barbosa GF, et al. Predicting the success of limited exploration for primary hyperparathyroidism using ultrasound, sestamibi, and intraoperative parathyroid hormone: analysis of 1158 cases. Ann Surg. 2008;248:420-8.

2. Udelsman R, Lin Z, Donovan P. The superiority of minimally invasive parathyroidectomy based on 1650 consecutive patients with primary hyperparathyroidism. Ann Surg. 2011;253:585-91.

3. The American Association of Clinical Endocrinologists and the American Association of Endocrine Surgeons position statement on the diagnosis and management of primary hyperparathyroidism. Endocr Pract. 2005;11:49-54.

4. Silverberg SJ, Shane E, Jacobs TP, Siris E, Bilezikian JP. A 10 -year prospective study of primary hyperparathyroidism with or without parathyroid surgery. N Engl J Med. 1999;341: 1249-55.

5. Udelsman R, Pasieka JL, Sturgeon C, Young JE, Clark OH. Surgery for asymptomatic primary hyperparathyroidism: proceedings of the Third International Workshop. J Clin Endocrinol Metab. 2009;94:366-72.

6. Zanocco K, Angelos P, Sturgeon C. Cost-effectiveness analysis of parathyroidectomy for asymptomatic primary hyperparathyroidism. Surgery. 2006;140:874-81.

7. Pinchot SN, Chen H. Non-operative management of primary hyperparathyroidism: the exception rather than the rule. Exp Rev Endocrinol Metab. 2009;4:553-63.

8. Greene $\mathrm{AB}$, Butler RS, McIntyre $\mathrm{S}$, et al. National trends in parathyroid surgery from 1998 to 2008: a decade of change. J Am Coll Surg. 2009;209:332-43.

9. Bilezikian JP, Khan AA, Potts JT Jr. Guidelines for the management of asymptomatic primary hyperparathyroidism: summary statement from the Third International Workshop. $J$ Clin Endocrinol Metab. 2009;94:335-9.

10. Cheung K, Wang TS, Farrokhyar F, Roman SA, Sosa JA. A metaanalysis of preoperative localization techniques for patients with primary hyperparathyroidism. Ann Surg Oncol. 2012;19:577-83.

11. Wang TS, Cheung K, Farrokhyar F, Roman SA, Sosa JA. Would scan, but which scan? A cost-utility analysis to optimize preoperative imaging for primary hyperparathyroidism. Surgery. 2011; 150:1286-94.

12. Rodgers SE, Hunter GJ, Hamberg LM, et al. Improved preoperative planning for directed parathyroidectomy with 4-dimensional computed tomography. Surgery. 2006;140:932-40.

13. Chen H, Sippel RS, Schaefer S. The effectiveness of radioguided parathyroidectomy in patients with negative technetium tc $99 \mathrm{~m}$ sestamibi scans. Arch Surg. 2009;144:643-8. 